\title{
INFECÇÃO HOSPITALAR NA PERSPECTIVA DOS PROFISSIONAIS DE ENFERMAGEM: UM ESTUDO BIBLIOGRÁFICO*
}

\author{
Luciana Borges Giarola ${ }^{1}$, Tatiane Baratieri², Andrea Monastier Costa ${ }^{3}$, João Bedendo ${ }^{4}$, Sonia Silva Marcon, \\ Maria Angélica Pagliarini Waidman ${ }^{6}$
}

\begin{abstract}
RESUMO: Estudo bibliográfico teve por objetivo identificar, na produção científica nacional, a forma como o profissional de enfermagem tem abordado o tema infecção hospitalar. Foram analisadas 15 publicações do período de dezanos - 1999 a 2009 -na base de dados SciELO; a busca foi feita no mês de março de 2010 e os dados analisados por meio da análise de conteúdo. Foram encontradas cinco categorias temáticas: o conceito de infecção hospitalar; a compreensão dos profissionais sobre infecção hospitalar e Comissão de Controle de Infecção Hospitalar; responsabilidade dos profissionais no controle de infecção hospitalar; a prevenção e controle da Infecção Hospitalar; e necessidade de formação adequada durante a graduação. Conclui-se que o tema deveria ser mais pesquisado, principalmente no que tange à Enfermagem e ao cuidado ao paciente; o presente estudo serve de subsídio para instigar discussões sobre o tema.

PALAVRAS-CHAVE: Infecção hospitalar; Equipe de enfermagem; Papel do profissional de enfermagem.

\section{HOSPITAL-ACQUIRED INFECTIONS FROM THE PERSPECTIVE OF NURSING PROFESSIONALS: A BIBLIOGRAPHICAL STUDY}

\begin{abstract}
This bibliographical study of Brazilian research output aimed to identify the way in which nursing professionals have approached the theme of hospital-acquired infections. Fifteen publications from the ten-year period 1999-2009 from the SciELO database were analyzed; the search was carried out in March 2010 and the data analyzed by content analysis. Five thematic categories were found: the concept of hospital-acquired infections; healthcareprofessionals' understanding of hospital-acquired infections and the Hospital-Acquired Infection Control Commission; healthcare professionals' responsibility in the control of hospital-acquired infections; the prevention and control of hospital-acquired infections; and thenecessityfor appropriate training during the undergraduate course. It is concluded that more research is needed, principally where nursing and patient care are affected. This study should serve as a base for discussion on the matter. KEYWORDS: Hospital-acquired infection; Nursing team; Role of the professional nurse.

\section{INFECCIÓN HOSPITALAR EN LA PERSPECTIVA DE LOS PROFESIONALES DE ENFERMERÍA: UN ESTUDIO BIBLIOGRÁFICO}

RESUMEN: Estudio bibliográfico cuyor objetivo fue identificar, en la producción científica nacional, la forma como el profesional de enfermería viene abordando el tema infección hospitalar. Fueron analizadas 15 publicaciones del periodo de diez años - 1999 a 2009 - en la base de datos SciELO; la búsqueda fue hecha en el mes de marzo de 2010 y los datos fueron analizados por medio del análisis de contenido. Surgieron cinco categorías temáticas: el concepto de infección hospitalar; la comprensión de los profesionales sobre infección hospitalar y Comisión de Control de Infección Hospitalar; responsabilidad de los profesionales en control de infección hospitalar; la prevención y control de Infección Hospitalar; y necesidad de formación adecuada durante la graduación. Se concluye que el tema debería ser más investigado, principalmente en lo que se refiere a la enfermería y al cuidado al paciente; el presente estudio sirve de subsidio para instigar discusiones sobre el tema.

PALABRAS-CLAVE: Infección hospitalar; Equipo de enfermería; Papel del profesional de enfermería.

\footnotetext{
*Artigo originado da disciplina Sistema de Cuidar do Programa de Pós-Graduação em Enfermagem da Universidade Estadual de Maringá - UEM.

${ }^{1}$ Enfermeira. Mestre em Enfermagem. Professora da Faculdade Ingá-UNINGÁ.

${ }^{2}$ Enfermeira. Mestre em Enfermagem. Professora do Departamento de Enfermagem da Universidade Estadual do Centro-Oeste.

${ }^{3}$ Enfermeira. Professora do Departamento de Enfermagem da Universidade Paranaense -UNIPAR. Mestranda pelo Programa de PósGraduação em Enfermagem da Universidade Federal do Paraná - UFPR.

${ }^{4}$ Enfermeiro. Doutor em Infectologia. Professor do Departamento e do Programa de Pós-Graduação em Enfermagem da UEM.

${ }^{5}$ Enfermeira. Doutora em Filosofia da Enfermagem. Professora do Departamento e do Programa de Pós-Graduação em Enfermagem e em Ciências da Saúde da UEM.

${ }^{6}$ Enfermeira. Doutora. Docente do Departamento e do Programa de Pós-Graduação em Enfermagem da UEM.
}

Autor correspondente:

Recebido: 19/07/2011

Luciana Borges Giarola

Aprovado: 09/01/2012

Faculdade Ingá

Rua Osvaldo Cruz, 317 - 87020-200 - Maringá-PR-Brasil

E-mail: lu_giarola@hotmail.com 


\section{INTRODUÇÃO}

As infecções hospitalares começaram a ocorrer na assistência em saúde a partir da criação de instituições destinadas a tratar os indivíduos, assim como pela implementação de procedimentos terapêuticos e diagnósticos progressivamente mais invasores ${ }^{(1)}$.

Segundo a Portaria n. 2616/98 do Ministério da Saúde, infecção hospitalar (IH) é aquela adquirida após admissão do paciente e que se manifeste durante a internação ou após a alta, quando relacionada com a internação ou a procedimentos hospitalares/ambulatoriais ou as manifestadas antes de 72 horas da internação, porém associadas a procedimentos diagnósticos e/ ou terapêuticos, realizados durante este período ${ }^{(2)}$. Os fatores de risco associados à aquisição de infecções, de um modo geral, estão relacionados ao próprio paciente, aos procedimentos invasivos e ao ambiente hospitalar ${ }^{(3)}$. Contudo, a probabilidade de o paciente adquirir uma infecção aumenta, na medida em que se utilizem equipamentos técnicos necessários ao seu tratamento, visto que tem possibilidade de romper suas defesas orgânicas $^{(4)}$. No Brasil, estima-se que $3 \%$ a $15 \%$ dos pacientes hospitalizados desenvolvem alguma $\mathrm{IH}^{(5)}$.

A história da medicina nos revela que as $\mathrm{IH}$ são tão antigas quanto a origem dos hospitais, sendo que as primeiras referências remontam o ano de 325 a.C., porém, somente em 1950 institui-se, na Inglaterra, a primeira Comissão de Controle de Infecção Hospitalar $(\mathrm{CCIH})^{(6)}$. A IH, na atualidade, representa preocupação não somente para os órgãos de saúde competentes, mas também um problema de ordem jurídica, social e ética em face às consequências na vida dos pacientes e o risco a que estes estão sujeitos ${ }^{(7)}$.

O papel da Enfermagem no controle da IH está presente desde suas primeiras descobertas. Florence Nightingale já apresentava preocupação com essa problemática e durante a Guerra da Criméia padronizou procedimentos de cuidados de enfermagem voltados à higiene e limpeza dos hospitais, introduzindo principalmente técnicas de anti-sepsia, com a finalidade de diminuir os riscos desse tipo de infecção ${ }^{(8)}$. A Enfermagem é a categoria profissional mais envolvida com os cuidados ao paciente, direta ou indiretamente, e, consequentemente, com a profilaxia e controle de infecções relacionada à assistência, em que a higiene das mãos tem um papel importante ${ }^{(9)}$.

Os profissionais que atuam em $\mathrm{CCIH}$, assim como aqueles que participam como membros da equipe de saúde e que deveriam realizar ações multidisciplinar- mente, sentem-se pela existência de muitas situações conflitantes entre a teoria e a prática, envoltos de dúvidas sobre o que fazer e como fazer para resolver questões que envolvem competência, compromisso, responsabilidade, honestidade, frente ao agir e pensar ético, considerando a individualidade, potencialidades, respeito e direitos do ser humano ${ }^{(10)}$.

A literatura aponta que uma das questões relativa ao tema pauta-se, especialmente, na responsabilidade dos profissionais e das instituições de saúde, com repercussões penais, civis e éticas. Isto porque, por vezes, a IH é decorrente de atos falhos cometidos pelos profissionais ${ }^{(7)}$. Contudo, estudo realizado com profissionais da área de saúde e do direito com o objetivo de apreender as representações sociais dos direitos dos usuários da saúde em casos de IH constatou a presença de ancoragens sociais, psicológicas e psicossociológicas, evidenciando preocupações com conotações positivas e negativas frente às infecções. A partir de comportamentos, até certo ponto, ambíguos, uma vez que eles reconhecem a necessidade de medidas preventivas, mas não as adota na proporção necessária. Além disso, reconhecem os direitos dos usuários mas não realizam ou realizam poucas atividades para garantir tais direitos ${ }^{(7)}$.

Diante do exposto, é de grande valia o conhecimento por parte dos profissionais da saúde sobre a importância de uma CCIH nas instituições, sendo indispensável que componentes das equipes multidisciplinares saibam das responsabilidades desta comissão, pois estão ligados direta e indiretamente aos cuidados com o paciente e tudo que o envolve, desde a qualidade do material escolhido até o mais complexo plano de cuidado traçado para o paciente internado.

A problemática do estudo se dá pelo fato de que a IH é uma questão de saúde pública e apresenta um caráter, na maioria das vezes, prevenível, tendo em vista que desde ações simples, como a lavagem das mãos, até ações mais complexas e a realização de uma cirurgia com a garantia de material adequadamente esterilizado. Envolve a ética e responsabilidade profissional para com suas ações, visando a manutenção/recuperação da saúde do outro. Neste sentido, a vigilância epidemiológica de IH é representada pela observação ativa, sistemática e contínua de sua ocorrência e distribuição entre pacientes, hospitalizados ou não, eventos e condições que afetam o risco e tem por objetivo a execução oportuna das ações de prevenção e controle ${ }^{(2)}$.

Ademais, este tipo de infecção resulta, além do ônus financeiro tanto para o paciente quanto para o

Cogitare Enferm. 2012 Jan/Mar; 17(1):151-7 
serviço público, prejuízos que ultrapassam tais questões, pois permeiam danos sociais e psicológicos. A necessidade de tratamentos medicamentosos, realização de inúmeros procedimentos, bem como o aumento no período de internação constituem exemplos de danos causados por ela. Outra problemática envolvida é a omissão do diagnóstico de IH, ações que ferem o direito do paciente e de sua família em ter ciência do seu estado de saúde. No que se refere a esta questão, o Instituto Brasileiro de Defesa do Consumidor assevera que há ausência de informação aos pacientes, o que se constitui em falha na abordagem do problema, pois desconsiderar os usuários como parte integrante da gestão de risco caracteriza deficiência técnica na estratégia de controle das infecções ${ }^{(11)}$.

Considerando a importância de se conhecer as possibilidades de oferecer cuidados a pacientes em potencial risco de infecção hospitalar ou aos que já tenham desenvolvido esta, a presente pesquisa teve como objetivo identificar na produção científica nacional a forma como os profissionais de enfermagem têm abordado o tema IH para estabelecer estratégias de cuidado de enfermagem.

\section{MÉTODO}

Trata-se de um estudo bibliográfico, o qual é entendido como aquele que explica um problema por meio de referências teóricas publicadas em documentos e, na maioria das vezes, busca compreender ou analisar as contribuições culturais ou científicas existentes sobre determinada temática. A busca se deu no mês de março de 2010, em que foram analisadas 15 publicações, sendo todos artigos completos, disponibilizados onli$n e$, divulgados na literatura nacional nos últimos dez anos. O corpus da análise foi composto pelo período de 1999 a 2009, correspondendo à produção científica encontrada sobre o tema no período especificado. A análise de conteúdo foi a técnica adotada para categorizar os dados, que significa classificar os elementos constitutivos de um conjunto por diferenciação e, em seguida, por reagrupamento segundo a analogia. As categorias, portanto são rubricas ou classes que reúnem um grupo de elementos com caracteres comuns em um título genérico ${ }^{(12)}$.

Na seleção dos artigos para análise foram utilizadas as seguintes palavras-chave: infecção hospitalar; equipe de enfermagem e enfermagem, e os seguintes critérios de inclusão: artigos com pelo menos um de seus autores enfermeiro; constar como texto completo nas bases de dados SciELO (Scientific Eletronic Library Online); ter sido publicado no período estipulado para o estudo e abordar o tema infecção hospitalar e cuidados de enfermagem.

Para a análise dos dados foi utilizado um roteiro contendo os dados de identificação do estudo (autores, tipo de estudo e principais resultados) assim como pontos norteadores a serem pesquisados: conceito de IH utilizado em cada artigo, pontos que relacionam profissionais de enfermagem, inclusive o cuidado e $\mathrm{IH}$, controle de infecção hospitalar ou CCIH.

\section{RESULTADOS}

Inicialmente foram encontrados 45 artigos completos na base de dados SciELO. Após análise dos resumos dos artigos obteve-se um total de 15 que se encaixavam nos critérios de inclusão, sendo 13 publicados em português e dois em inglês, os quais, após leitura do texto completo, foram utilizados para a pesquisa.

Do processo de análise dos artigos encontrados emanaram cinco eixos temáticos, incluindo o conceito de $\mathrm{IH}$, o entendimento dos profissionais sobre o termo, assim como sobre $\mathrm{CCIH}$, destacando a atuação do enfermeiro, além da responsabilidade dos profissionais de saúde frente ao controle da infecção hospitalar e sua prevenção, bem como a necessidade da adequação da grade curricular no que tange ao tema em questão.

\section{DISCUSSÃO}

\section{O conceito de infecção hospitalar}

O termo IH é discutido por vários autores, porém dos artigos analisados, a maioria ${ }^{(13-18)}$, utiliza o conceito determinado pela Portaria n. 2.616 de 12 de maio de $1998^{(2)}$. Os autores salientam tratar-se de uma infecção adquirida após a internação do paciente e que se manifesta durante a mesma ou após a alta quando puder ser relacionada com a internação ou procedimentos hospitalares. Acredita-se que esse é o conceito que melhor define o termo e pode servir de base para estruturar discussões sobre o tema.

A IH é abordada também de forma resumida, sendo considerada como qualquer processo infeccioso adquirido no ambiente hospitalar ${ }^{(19)}$, se constituindo em risco à saúde dos pacientes do serviço que se submetem a 
procedimentos terapêuticos ou de diagnóstico ${ }^{(20)}$. Além disso, pode-se afirmar que a IH constitui iatrogenia decorrente da hospitalização e que se torna importante foco de atenção nas últimas décadas, embora desde a Antiguidade existissem relatos sobre a disseminação de doenças epidêmicas e sobre a inevitabilidade das infecções cirúrgicas ${ }^{(17)}$.

Com essas explanações nota-se que a IH é um processo infeccioso adquirido no ambiente hospitalar e, na maioria das vezes, sua ocorrência se dá por fatores evitáveis, por exemplo, pela lavagem inadequada das mãos, manuseio de materiais e realização de técnicas desrespeitando os princípios de assepsia e falta de controle rigoroso no processamento dos materiais esterilizados, desde a lavagem até armazenamento e distribuição dos mesmos.

$\mathrm{Na}$ atualidade, destaca-se que a IH representa uma preocupação, não somente dos órgãos de saúde competentes, mas principalmente é considerada um problema de ordem social, ética e jurídica em face às implicações na vida dos pacientes e o risco que estão submetidos ${ }^{(18)}$. Autores também ressaltam que a instituição das precauções padrão (uso dos equipamentos de proteção individual, higienização das mãos e manuseio adequado de materiais perfurocortantes), têm como objetivo a minimização dos casos de infecção, tiveram início com a epidemia da Aids e para muitos profissionais o foco de preocupação é a infecção pelo HIV ${ }^{(19)}$.

\section{A compreensão dos profissionais sobre IH e CCIH: atuação do enfermeiro}

No que tange ao entendimento dos profissionais de saúde sobre a IH, os estudos revelam que seu controle se dá por meio, principalmente, da paramentação adequada da execução de técnicas e da lavagem de mãos ${ }^{(14,16-18,20-22)}$.

Nesse sentido, autores defendem que a IH está associada à contaminação, em especial de materiais e equipamentos hospitalares, portanto estes meios devem ser valorizados como determinantes das infecções hospitalares em detrimento de medidas fundamentais de controle e prevenção, como a lavagem das mãos e uso de equipamentos de proteção individual ${ }^{(23)}$. Por outro lado, autores expõem que para prevenir a IH os profissionais e estudantes da área da saúde valorizam a paramentação adequada do executor do procedimento, além de ressaltar a importância do cuidado com as mãos ${ }^{(18)}$.

Em pesquisa realizada em um hospital de Goiânia chamou a atenção o fato de ter sido observado adesão ao uso das Precauções Padrão somente para os casos de atendimento a pacientes com diagnósticos definidos, em especial nos casos de doenças transmissíveis e infecciosas. Este tipo de adesão torna vulnerável a segurança dos envolvidos no processo assistencial e facilita o desenvolvimento dos casos de IH entre os assistidos. Esta adesão revela uma concepção errada embasada nas crenças dos indivíduos de que os riscos existem, mas em situações concretas. Além disso, há que se pensar na possibilidade de estarmos convivendo com outros microrganismos, de importância epidemiológica ainda desconhecida, cujos agravos não podemos dimensionar ${ }^{(22)}$.

Pesquisa realizada em Portugal ${ }^{(16)}$ revela que os enfermeiros daquele país entendem a IH como um riscoao qual pacientes e profissionais estão expostos no ambiente hospitalar, e que sua prevenção depende de procedimentos referentes ao isolamento de pacientes e a lavagem das mãos. $O$ estudo constatou também que os enfermeiros são vistos como cuidadosos e responsáveis, e que a IH tem ocorrido de maneira proporcional ao desenvolvimento da tecnologia invasiva, porém o conhecimento dos profissionais da saúde sobre as ações de prevenção e controle das infecções não tem acompanhado este desenvolvimento ${ }^{(16)}$. É preocupante saber que na concepção de muitos profissionais o controle da IH é de responsabilidade das $\mathrm{CCIH}^{(13)}$, uma vez que o profissional está se isentando de culpa. No entanto, em muitos casos o controle das infecções é inerente à prática profissional, considerando os riscos na manipulação de matéria orgânica, secreções e excreções, assim como o contato com microrganismos disseminados por diferentes vias de transmissão ${ }^{(14)}$.

Sobre a CCIH, estudo revela que muitos profissionais de saúde sequer sabem o que significa a referida sigla, além de não conhecer os profissionais que nela atuam ${ }^{(14)}$. O papel da referida comissão é normatizar os procedimentos e condutas a partir de uma vigilância epidemiológica contínua ${ }^{(15)}$, além disso, é de sua competência elaborar um Programa de Controle de Infecções Hospitalares (PCIH), com ações sistematizadas, com vistas a reduzir a incidência e gravidade das $\mathrm{IH}^{(24)}$. Outro ponto relevante é que o enfermeiro participa da CCIH muitas vezes tem sobrecarga de trabalho, desempenhando duplo papel na instituição, ou seja, atua na comissão ao mesmo tempo ocupa outro cargo, o que prejudica o desenvolvimento de ações adequadas no campo do controle de infecção ${ }^{(10)}$.

A CCIH também é vista por muitos profissionais como uma comissão importante para analisar e apurar os responsáveis por casos de $\mathrm{IH}^{(16)}$. A educação per- 
manente também é assumida por esta comissão, na tentativa de encontrar meios que promovam mudanças mais duradouras e eficazes referentes à diminuição e controle da IH. No entanto, sob um outro olhar, a adoção a certas medidas de prevenção é um ato voluntário e individual que depende da decisão de cada profissional. Desta forma, é influenciada, dentre outros fatores, pela complexidade inerente ao profissional que presta o cuidado ${ }^{(20)}$.

As pesquisas analisadas apontam que para a redução nos índices de IH é necessário investir em capacitação dos profissionais, cabendo ao enfermeiro oferecer educação continuada a sua equipe ${ }^{(14-15,17,19,21,24)}$.

É importante a participação do enfermeiro no processo de prevenção da $\mathrm{IH}$, tendo em vista que pode ser considerado um elo entre os profissionais. Além disso, o enfermeiro precisa estar integrado com a CCIH, assim como é preciso comprometimento e envolvimento de todas as partes, tanto dos profissionais que estão diretamente em contato com o paciente, assim como das chefias, por meio da viabilização de recursos humanos e materiais ${ }^{(15,24)}$.

Nesse sentido, é importante ressaltar que a falta de uma política de educação permanente para os profissionais tem contribuído para dificultar, sensivelmente, a conquista da realização integral, igualitária e universal dos direitos sociais na saúde, tornando inquestionável a necessidade de uma política pública de saúde ${ }^{(21)}$. Um dos estudos analisados revela que existe carência de informações básicas para os profissionais aderirem às medidas de prevenção e controle de IH, consideradas deficitárias no que se refere à formação e orientação para o seu controle pelas instituições formadoras; ações educativas desenvolvidas pela CCIH; observância com o cuidado da pele e lavagem das mãos; a necessidade de investimentos efetivos envolvidos neste processo e a persistência da prática medicamentosa para solucionar o problema da infecção com antibióticos ${ }^{(21)}$.

\section{Responsabilidade dos profissionais no controle de infecção hospitalar}

A principal responsabilidade dos profissionais de saúde se dá frente às questões éticas, ressaltando que muitas vezes o interesse da instituição é sobreposto aos direitos do paciente, infringindo os postulados do Código de Ética, norteador da profissão e de seus direitos. Em muitos casos, os profissionais da área de saúde não têm preocupação com os deveres éticos e jurídicos que lhes possam responsabilizar pelo exercício de sua profissão. Prova disso são os muitos casos de omissões, assim como a ocorrência de imperícia, negligência, ou imprudência, pela representação político-social das infecções hospitalares e de seu controle ${ }^{(10,21)}$.

O profissional enfermeiro é visto como o principal responsável pelo papel educativo de toda a equipe de saúde, considerando o melhor vínculo com a equipe, assim como sua supervisão contínua, tendo como funções planejar, implementar e participar dos programas de formação, qualificação contínua e promoção da saúde dos trabalhadores ${ }^{(13,23-24)}$.

Para que as medidas preventivas sejam tomadas também é necessário que os gestores das instituições estejam cientes da problemática, uma vez que eles não prestam um cuidado direto ao paciente, porém fornecem subsídios para a prática acontecer e são responsáveis também pela realização de um bom trabalho no ambiente hospitalar, prevenindo e controlando as infecções de forma indireta ${ }^{(16,22)}$.

Considera-se o controle de IH de responsabilidade de todos os profissionais de saúde que prestam assistência direta ao paciente ${ }^{(15)}$. Entretanto, a realidade que se apresenta é de que as pessoas trabalham de forma individualizada frente a essa questão, fragmentando e descontextualizando a assistência prestada ${ }^{(10)}$. Contudo, quando, na nossa subjetividade, acreditamos que as nossas ações são o bem, mesmo que a finalização não seja, o sentimento do dever cumprido e a satisfação de uma resposta imediata às necessidades do paciente, com desfecho positivo/negativo, suplantam os sentimentos decorrentes das falhas durante o processo, onde a avaliação de tal fato não é vista como necessária. Além disso, avaliar situações de erros remete o indivíduo à condição de transgressor de normas, protocolos, valores, entre outros ${ }^{(22)}$.

$\mathrm{O}$ cuidado de enfermagem quando se refere ao profissional que atua em CCIH envolve questões além daquelas da prática diária da assistência da IH. Entrelaça questões ontológicas e epistemológicas da Enfermagem, abarca o ser que cuida e é cuidado, envolve a ética da enfermagem, a arte propriamente dita, questões importantes, geralmente esquecidas no dia a dia do enfermeiro - que geralmente está preocupado com o gerenciamento de atividades e no cumprimento de normas e rotinas. $\mathrm{O}$ enfermeiro que atua na $\mathrm{CCIH}$ precisa estar atento ao que rodeia a vida do outro, e esse outro não é somente o doente, é a equipe, a família e todos que adentram a instituição de saúde, podendo levar e trazer microorganismos que prejudicam a vida daqueles que estão mais vulneráveis. Neste sentido, os enfermeiros preocupados 
com a IH precisam, constantemente, fazer atividades de educação continuada.

\section{Formação adequada durante a graduação}

Estudos apontam para a necessidade da inserção do tema IH nas grades curriculares ${ }^{(10,15,23)}$. As instituições de ensino superior têm responsabilidades pela formação de profissionais competentes, e as escolhas das práticas pedagógicas determinam a atividade do profissional. Em muitos casos, os conteúdos que abordam a temática são insuficientes para uma atuação efetiva, ou seja, falta conhecimento, clareza e visão ampliada de prevenção ${ }^{(24)}$. Desta forma, a instituição de ensino deve compor-se de um corpo docente comprometido com essas questões, atendendo aos princípios do exercício profissional, em uma estrutura organizacional e funcional que favoreça a prática de condutas $\operatorname{corretas}^{(16,21)}$. Existe uma dubiedade no que tange a abordagem da prevenção e controle da IH durante a graduação, ou seja, alguns acreditam que o tema deva estar em disciplina específica, e outros afirmam que a melhor forma de oferecer tal conteúdo é durante todo o curso, permeando cada disciplina ou especialidade ${ }^{(25)}$.

Vale salientar que intervir na formação profissional é interferir no momento em que está sendo elaborado o conhecimento e as habilidades técnicas estão sendo desenvolvidas pelo indivíduo. Assim, durante a graduação é o melhor momento para formar uma consciência crítica acerca do controle de infecção nos futuros profissionais ${ }^{(13)}$.

\section{CONSIDERAÇÕES FINAIS}

Os estudos revelam que a equipe de enfermagem é apontada como a principal responsável pela prevenção e CIH. Percebe-se que para um controle adequado da IH é fundamental a paramentação correta e a realização de técnicas assépticas. Um ponto de destaque é a necessidade da formação profissional adequada, a qual é fundamental para desenvolver uma consciência crítica e compromissada com o controle da IH.

A partir da análise destes estudos é possível inferir que ainda existe uma lacuna no que tange ao cuidado de enfermagem e a atuação do enfermeiro na CCIH, pois as demandas vão além das atividades administrativas, envolvem questões éticas, sociais, humanas e de direitos dos pacientes e dos trabalhadores havendo a necessidade de atuação em serviço como uma realidade permanente.

Com a busca bibliográfica percebeu-se que ainda são escassos os estudos realizados por enfermeiros que abordam essa temática. Considera-se que o assunto deveria ser amplamente discutido e enfatizado, não apenas em ambientes específicos e de maior vulnerabilidade, por exemplo o hospital, mas no sistema de saúde como um todo e na formação dos profissionais de saúde.

Nesse âmbito, como estratégia para melhorar o cuidado de enfermagem ao paciente, pode-se pensar em uma formação com maior ênfase no tema IH, constante capacitação/conscientização dos profissionais frente à prevenção e controle de infecções, além da ética que envolve a questão e cada categoria profisisonal, com a ciência de que todos devem prezar pela vida do paciente, executando ações com responsabilidade, livre de danos decorrente de negligência, imperícia e imprudência.

Esse estudo abre as portas para uma gama de discussões no âmbito da infecção hospitalar, tornandose um leque para a realização de inúmeros estudos que contemplem os vários aspectos que envolvem a temática, especialmente por parte dos profissionais de enfermagem, pois são os que passam mais tempo na instituição de saúde e realizam a maior parte dos procedimentos junto aos pacientes.

\section{REFERÊNCIAS}

1. Cruz EDA. Resgatando a autoria da equipe de saúde no planejamento de ações de prevenção de infecções hospitalares. Cogitare enferm. 1996;1(2):26

2. Brasil. Portaria n. 2.616, de 12 de maio de 1998. Regulamenta as ações de controle de infecções hospitalares no Brasil. Gabinete do Ministro, Brasília. 12 maio 1998.

3. Arantes A, Carvalho ES, Medeiros EAS, Farhat CK, Mantese OC. Uso de diagramas de controle na vigilância epidemiológica das infecções hospitalares. Rev. saude publica. 2003;37(6):768-74.

4. Bare SC, Smeltzer BG. Tratado de enfermagem médicocirúrgico. 10ª ed. Rio de Janeiro: Guanabara; 2006.

5. Scheidt KLS, Carvalho M. Avaliação prática da lavagem das mãos pelos profissionais de saúde em atividades lúdico-educativas. Rev. bras. enferm. 2006;14(4):221-5.

6. Martins MA. Manual de infecção hospitalar: epidemiologia, prevenção e controle. $2^{\mathrm{a}}$ ed. Rio de Janeiro: Médica e Científica Ltda; 2005. 
7. Sousa CMM, Alves MSCF, Moura MEB, Silva AO. Os direitos dos pacientes da saúde em casos de infecção hospitalar. Rev. bras. enferm. 2008;61(4):411-7.

8. Fontana RT. As infecções hospitalares e a evolução das infecções. Rev. bras. enferm. 2006;58(5):703-6.

9. Tripple AFV, Mendonça KM, Melo MC, Silva e Souza AC, Pereira MS, Santos SLV. Higienização das mãos: o ensino e a prática entre graduandos na área da saúde. Acta sci. health sci. 2007;29(2):107-14.

10. Alves DCI, Évora YDM. Questões éticas envolvidas na prática profissional de enfermeiros da comissão de controle de infecção hospitalar. Rev. latino-am. enfermagem. 2002;10(3):265-75.

11. IDEC. Instituto Brasileiro de Defesa do Consumidor. O Controle de Infecção Hospitalar no Brasil e os Consumidores. São Paulo, 2006. Disponível em: http:// www.idec.org.br/arquivos/relatorio_IH.pdf. Acesso em 2011 set 20 .

12. Bardin L. Análise de conteúdo. Lisboa: Edições 70; 2008.

13. Pereira MS, Souza ACS, Tipple AFV, Prado MA. A infecção hospitalar e suas implicações para o cuidar da enfermagem. Texto contexto enferm. 2005;14(2):250-7.

14. Oliveira AC, Evangelista S, Lucas TC, Mourão PHO, Clemente WT. A percepção da equipe multiprofissional sobre a comissão de controle de infecção hospitalar. Online braz. j. nurs. [Internet] 2006;5(2) [acesso em 25 jun 2011]. Disponível: http://bases.bireme.br/cgi-bin/ wxislind.exe/iah/online/?IsisScript $=$ iah/iah.xis\&src $=$ google \&base $=$ LILACS\&lang $=$ p\&nextAction $=1 n k \& e$ xprSearch $=490090 \&$ indexSearch=ID

15. Fontana RT, Lautert L. A prevenção e o controle de infecções: um estudo de caso com enfermeiras. Rev. bras. enferm. 2006;59(3):257-61.

16. Moura MEB, Ramos MN, Sousa CMM, Silva AO, Alves, MSCF. Infecção hospitalar no olhar de enfermeiros portugueses: representações sociais. Texto contexto enferm. 2008;17(4):743-9.

17. Turrini RNT. Percepção das enfermeiras sobre fatores de risco para a infecção hospitalar. Rev. esc. enferm. USP. 2000;34(2):174-84.

18. Moura MEB, Tapety FI, Carvalho CMRS, Oliveira JNP, Matos FTC, Moura LKB. Representações sociais das infecções hospitalares elaboradas pelos profissionais de saúde. Rev. bras. enferm. 2008;61(4):418-22.

19. Kunzle SEM, Pereira CS, Alves KC, Pelá NTR, Gir E. Auxiliares e técnicos de enfermagem e controle de infecção hospitalar em centro cirúrgico: mitos e verdades. Rev. esc. enferm. USP. 2006;40(2):214-20.

20. Neves ZCP, Tipple AFV, Souza ACS, Pereira MS, Melo DS, Ferreira LR. Hand hygiene: the impact of incentive strategies on adherence among healthcare workers from a newborn intensive care unit. Rev. latino-am. enfermagem. 2006;14(4):546-52.

21. Sousa CMM, Feitosa MS, Moura MEB, Silva AO. Representações sociais das implicações legais da infecção hospitalar e de seu controle. Rev. bras. enferm. 2007;60(4,):428-33.

22. Melo DS, Silva E, Souza AC, Tipple AFV, Neves ZCP, Pereira MS. Nurses' understanding of standard precautions at a public hospital in Goiania-GO, Brazil. Rev. latino-am. enfermagem. 2006;14(5):720-7.

23. Santos AMR, Cabral LAF, Brito DS, Madeira MZA, Silva MEDC, Martins MCC. As representações sociais da infecção hospitalar elaboradas por profissionais de enfermagem. Rev. bras. enferm. 2008;61(4):441-6.

24. Cucolo DF, Faria JIL, Cesarino CB. Avaliação emancipatória de um programa educativo do serviço de controle de infecção hospitalar. Acta paul. enferm. 2007;20(1):49-54.

25. Tipple AFV, Pereira MS, Hayashida M, Moriya TM, Souza ACS. O ensino do controle de infecção: um ensaio teórico-prático. Rev. latino-am. enfermagem. 2003;11(2):245-50. 